\title{
Collective oscillations of an interacting trapped Fermi gas
}

\author{
L. Vichi and S. Stringari \\ Dipartimento di Fisica, Università di Trento and Istituto Nazionale per la Fisica della Materia, I-38050 Povo, Italy
}

(May 12, 1999)

We calculate the effects of two-body interactions on the low frequency oscillations of a normal Fermi gas confined in a harmonic trap. The mean field contribution to the collective frequencies is evaluated in the collisionless regime using a sum rule approach. We also discuss the transition between the collisionless and hydrodynamic regime with special emphasis to the spin dipole mode in which two atomic clouds occupying different spin states oscillate in opposite phase. The spin dipole mode is predicted to be overdamped in the hydrodynamic regime. The relaxation time is calculated as a function of temperature and the effects of Fermi statistics are explicitly pointed out.

05.30.Fk, 51.10+y, 32.80.Pj, 67.55.Jd

The investigation of collective excitations in trapped atomic gases has become an active research field, stimulated by the experimental realization of Bose-Einstein condensation 近. Because of the high density of the condensate, interaction effects in cold Bose gases are crucial [2] in order to explain the experimental results for the collective frequencies [3]. In Fermi gases the Pauli exclusion principle makes the density of the trapped gas more dilute, thereby reducing the effects of interactions. The equilibrium properties of trapped Fermi gases as well as of Fermi-Bose mixtures have been already the object of theoretical calculations based on mean field approaches [1] [7. On the other hand experiments aiming to produce samples of Fermi gases in conditions of quantum degeneracy are also becoming available [8].

In this letter we provide a theoretical discussion of interaction effects on the collective oscillations of a trapped Fermi gas. The very high precision of frequency measurements, the large value of the scattering length exhibited by some atomic species and the possibility of pointing out effects of quantum statistics in the collisional term, make the study of collective oscillations in these systems a promising area of investigation. We will consider Fermi gases occupying two distinct spin states (hereafter called, for simplicity, spin up and spin down respectively). In fact only in this case can the effects of the interaction generated by $s$-wave scattering be explored. We will further limit the discussion to the case of excitations of low multipolarity in the normal phase, corresponding to the easiest realization of future experiments. In fact the transition to the superfluid phase is predicted [9, 10] to occur at very low temperatures. For a discussion of the oscillations in the superfluid phase see [10,11].

At very low temperatures collisions are quenched by
Fermi statistics and the system is in the collisionless regime, corresponding to the propagation of zero sound in traditional Fermi liquids 12. A useful approach to the study of collective excitations in this regime is provided by sum rules [13. In this approach the excitation energies are estimated through the ratio

$$
\hbar \omega=\sqrt{\frac{m_{3}}{m_{1}}}
$$

between the $k=3$ and $k=1$ moments $m_{k}=$ $\int S(E) E^{k} d E$ of the dynamic structure factor

$$
S(E)=\sum_{n}|\langle n|F| 0\rangle|^{2} \delta\left(E-\hbar \omega_{n}\right)
$$

relative to a given operator $F$. For simplicity we have considered the $T=0$ case, but the formalism can be naturally extended to finite temperature. The moments $m_{1}$ and $m_{3}$ are easily evaluated in terms of commutators. In fact, using the completeness relation, one can write

$$
\begin{aligned}
& m_{1}=\frac{1}{2}\left\langle 0\left|\left[F^{\dagger},[H, F]\right]\right| 0\right\rangle, \\
& m_{3}=\frac{1}{2}\left\langle 0\left|\left[\left[F^{\dagger}, H\right],[H,[H, F]]\right]\right| 0\right\rangle,
\end{aligned}
$$

thereby avoiding the most difficult problem of determining the full function $S(E)$. In eq.(3-4) $|0\rangle$ is the ground state of the many body system. Let us consider a symmetric configuration with $N_{\uparrow}=N_{\downarrow}=N / 2$ and the same confining potential $V_{e x t}^{\uparrow}=V_{e x t}^{\downarrow}=V_{e x t}$ for the two spin species. We will evaluate the commutators using the mean field hamiltonian

$$
H=\sum_{i} \frac{p_{i}^{2}}{2 m}+V_{e x t}+g \sum_{i \uparrow, j \downarrow} \delta\left(\mathbf{r}_{i}-\mathbf{r}_{j}\right)
$$

where $g=4 \pi \hbar^{2} a / m$ is the interaction coupling constant fixed by the $s$-wave scattering length $a$. By choosing an isotropic harmonic potential $V_{\text {ext }}(r)=\frac{1}{2} m \omega_{h o}^{2} r^{2}$ the commutators with the monopole $\left(F=\sum_{i} r_{i}^{2}\right)$, quadrupole $\left(F=\sum_{i} r_{i}^{2}-3 z_{i}^{2}\right)$ and dipole $\left(F=\sum_{i} z_{i}\right)$ operators can be easily evaluated and one obtains the following results 14

$$
\begin{gathered}
\omega_{M}=\sqrt{\frac{2 E_{k i n}+6 E_{h o}+6 E_{i n t}}{N m\left\langle r^{2}\right\rangle}}=2 \omega_{h o} \sqrt{1+\frac{3}{8} \frac{E_{i n t}}{E_{h o}}}(6) \\
\omega_{Q}=\sqrt{\frac{E_{k i n}+E_{h o}}{N m\left\langle r^{2}\right\rangle}}=2 \omega_{h o} \sqrt{1-\frac{3}{4} \frac{E_{i n t}}{E_{h o}}}
\end{gathered}
$$




$$
\omega_{D}=\omega_{h o}
$$

where $E_{\text {int }}=g \int \rho(r)^{2} d \mathbf{r} / 4$ is the mean field interaction energy, $E_{h o}=m \omega_{h o}^{2} \int r^{2} \rho(r) d \mathbf{r} / 2$ is the potential oscillator energy, $E_{k i n}=\sum_{i}\left\langle 0\left|p_{i}^{2} / 2 m\right| 0\right\rangle$ is the kinetic energy relative to the ground state and $\rho=\rho_{\uparrow}+\rho_{\downarrow}$ is the total density of the gas. In deriving the second equalities in (6-7) we have used the virial theorem [2]

$$
2 E_{k i n}-2 E_{h o}+3 E_{\text {int }}=0
$$

which allows one to calculate the deviations of the collective frequencies from the ideal gas prediction $2 \omega_{h o}$ in terms of the ratio $E_{\text {int }} / E_{h o}$. Differently from the quadrupole and the monopole, the dipole frequency is not affected by interactions. In fact this mode corresponds to the oscillation of the center of mass of the gas driven by the external harmonic potential. It is worth noticing that results (6-8) hold also in the case of trapped bosons 14], provided one uses the corresponding expression for the interaction energy. In the case of BoseEinstein condensed gases the ground state kinetic energy is strongly quenched by interactions and, for large $N$, the ratio $E_{\text {int }} / E_{\text {ho }}$ is equal to $2 / 3$ (see eq.(9)). In this limit one recovers the results $\omega_{M}=\sqrt{5} \omega_{h o}$ and $\omega_{Q}=\sqrt{2} \omega_{h o}$ predicted by the hydrodynamic theory of superfluids 14 . Conversely, in the Fermi case the interaction energy is, in most cases, only a small perturbation that can be safely estimated using the Thomas-Fermi expression [15]

$$
\rho(r)=\left(\frac{2 m}{\hbar^{2}}\right)^{3 / 2} \frac{1}{3 \pi^{2}}\left(\mu_{0}-V_{e x t}(r)\right)^{3 / 2}
$$

for the ground state density. In this equation $\mu_{0}=$ $(3 N)^{1 / 3} \hbar \omega_{h o}$ is the chemical potential of the Fermi gas, fixed by the normalization condition. Using expression (10) for the density one obtains the result

$$
\frac{E_{\text {int }}}{E_{\text {ho }}}=\alpha \frac{N^{1 / 6} a}{a_{h o}}
$$

where $\alpha=8192 \sqrt{2} 3^{1 / 6} / 2835 \pi^{2} \simeq 0.50$ while $a_{h o}=$ $\left(\hbar / m \omega_{h o}\right)^{1 / 2}$ is the usual harmonic oscillator lenght. Interaction effects are governed by the combination $N^{1 / 6} a / a_{h o}$ showing that in order to emphasize the role of interactions it is much more efficient to increase the ratio $a / a_{h o}$ rather than the number of atoms. The ratio (11) can be also casted in the form $E_{\text {int }} / E_{h o} \simeq 0.3 \times k_{F} a$ where $k_{F}=\left(2 m(3 N)^{1 / 3} \omega_{h o} / \hbar\right)^{1 / 2}$ is the Fermi momentum of the trapped gas 15 .

In analogous way one can calculate the frequency of the out of phase oscillations, hereafter called spin excitations. We report here the result for the most relevant spin dipole mode excited by the operator $F=\sum_{i \uparrow} z_{i}-\sum_{i \downarrow} z_{i}$. This mode corresponds to a relative oscillation of the centers of mass of the spin-up and spin-down clouds and is the analog of the giant dipole resonance exhibited by atomic nuclei [16]. The frequency of the spin dipole mode is found to be

$$
\begin{aligned}
\omega_{S D}=\sqrt{\omega_{h o}^{2}-\frac{g}{m N} \int\left|\partial_{z} \rho\right|^{2} d \mathbf{r}} & \\
& \simeq \omega_{h o}\left(1-\alpha^{\prime} \frac{N^{1 / 6} a}{a_{h o}}\right)
\end{aligned}
$$

where $\alpha^{\prime}=128 \sqrt{2} 3^{1 / 6} / 35 \pi^{2} \simeq 0.63$ and, in the second equality, we have used the ground state density (10) to evaluate the interaction contribution to first order in the scattering length. Notice that interactions affect the spin-dipole mode through the same combination of parameters characterizing the ratio (11). Typical values for the fermionic isotope of potassium show that interaction effects are rather small. For example, using $N=5 \times 10^{5}$ and $a / a_{h o}=6 \times 10^{-3}$ one finds that the dipole frequency decreases by $\sim 3 \%$. Significantly larger corrections are predicted in the case of ${ }^{6} \mathrm{Li}$ where the scattering length is a factor 10 larger and negative. Notice that the integrals characterizing the interaction contribution to the collective frequencies, are calculated here at $T=0$ and would decrease at higher temperature. We also note that, at $T=0$, sum rules provide only an upper bound to the frequency of the lowest state excited by the operator $F$. However, if one considers first order corrections in the interaction constant $a$, the sum rule bounds $(6-8,12)$ can be shown to correspond to the exact value of the collective frequencies.

The results derived above can be easily generalized to the case of deformed traps in which the external potential reads $V_{\text {ext }}(\mathbf{r})=m \omega_{\perp}^{2}\left(x^{2}+y^{2}+\lambda^{2} z^{2}\right) / 2$ and $\lambda=\omega_{z} / \omega_{\perp}$ is the deformation parameter of the trap. By assuming that interaction effects are smaller than the unperturbed splitting between the radial and axial frequencies, one finds simple results also in this case. The new decoupled frequencies, associated with the radial and axial excitation operators $F_{\text {radial }}=\sum_{i} x_{i}^{2}+y_{i}^{2}$ and $F_{\text {axial }}=\sum_{i} z_{i}^{2}$, become, to first order in $a, \omega_{\text {radial }}=2 \omega_{\perp}$ and $\omega_{\text {axial }}=2 \omega_{z}\left(1-3 / 16 \alpha N^{1 / 6} a / a_{h o}\right)$ showing that only the axial mode is affected by the interaction. The oscillator length $a_{h o}=(\hbar / m \bar{\omega})^{1 / 2}$ is here defined in terms of the geometrical average $\bar{\omega}=\omega_{\perp} \lambda^{1 / 3}$ of the three frequencies. For the spin dipole mode result (12) is easily generalized to both the radial and axial directions. One finds $\omega_{\text {radial }}=\omega_{\perp}\left(1-\alpha^{\prime} N^{1 / 6} a / a_{h o}\right)$ and $\omega_{\text {axial }}=\omega_{z}\left(1-\alpha^{\prime} N^{1 / 6} a / a_{h o}\right)$.

So far we have ignored the effects of collisions. If the collisional frequency is much larger than the frequency of the collective excitations then the system is in the hydrodynamic regime, also known as first sound regime. An important question is whether the transition between the collisionless and hydrodynamic regimes takes place in the degenerate or classical regime for our trapped gases. Let us first discuss the collective frequencies of a trapped Fermi gas in the full hydrodynamic regime (see 17 for a recent discussion). In the spherical case the quadrupole frequency becomes $\omega_{Q}^{H D}=\sqrt{2} \omega_{h o}$ instead of (7). This 
result is independent of statistics and holds also for a classical gas [18]. The reduction with respect to the collisionless value is due to the fact that, in the collisional regime, the only restoring force for surface excitations arises from the external field. For the monopole frequency one instead finds that result (6) holds also in the hydrodynamic regime. The above analysis can be also extended to the case of deformed traps [17] where one finds that, in the absence of mean field effects, the collective frequencies coincide with the ones holding for a classical gas [18].

Collisions are expected to have more dramatic consequences on the spin-dipole oscillation since they do not conserve the spin current and consequently give rise, in the hydrodynamic regime, to a pure diffusive mode. The investigation of the spin dipole mode is consequently expected to be a sensitive test of the role of collisions and, possibly, of quantum statistics. The equations for the spin dipole oscillation can be easily obtained starting from the Boltzmann equation where the effects of Fermi statistics are included in the collisional integral. For a first estimate let us ignore the mean field effect, which is responsible for the frequency shift of (12). Using the method of the averages recently developed in 20] for classical trapped gases, one obtains the following coupled equations:

$$
\begin{aligned}
& \partial_{t}\left\langle z_{\uparrow}-z_{\downarrow}\right\rangle-\left\langle v_{z \uparrow}-v_{z \downarrow}\right\rangle=0 \\
& \partial_{t}\left\langle v_{z \uparrow}-v_{z \downarrow}\right\rangle+\omega_{z}^{2}\left\langle z_{\uparrow}-z_{\downarrow}\right\rangle=\left\langle\left(v_{z \uparrow}-v_{z \downarrow}\right) I_{c o l l}\right\rangle
\end{aligned}
$$

where the average $\langle\ldots\rangle$ is taken both in coordinate and momentum space. The collisional term is given by

$$
\begin{gathered}
\left\langle\left(v_{z \uparrow}-v_{z \downarrow}\right) I_{\text {coll }}\right\rangle= \\
-\frac{\sigma m^{6}}{4 \pi h^{6}} \frac{2}{N} \int d \mathbf{r} d \mathbf{v}_{1} d \mathbf{v}_{2} d \Omega\left|\mathbf{v}_{1}-\mathbf{v}_{2}\right|\left(v_{1 z}-v_{2 z}\right) \times \\
\times\left[\left(1-f_{1 \uparrow}\right)\left(1-f_{2 \downarrow}\right) f_{1 \uparrow}^{\prime} f_{2 \downarrow}^{\prime}-f_{1 \uparrow} f_{2 \downarrow}\left(1-f_{1 \uparrow}^{\prime}\right)\left(1-f_{2 \downarrow}^{\prime}\right)\right]
\end{gathered}
$$

where $\sigma=4 \pi a^{2}$ is the total cross section, $\mathbf{v}_{1}^{\prime}$ and $\mathbf{v}_{2}^{\prime}$ are the velocities of the particles 1 and 2 after the collision, and $f_{\uparrow}, f_{\downarrow}$ are the distribution functions relative to the two spin components, normalized to $(\mathrm{m} / h)^{3} \int f_{\uparrow} d \mathbf{r} d \mathbf{v}=$ $(m / h)^{3} \int f_{\downarrow} d \mathbf{r} d \mathbf{v}=N / 2$. The collisional term can be estimated by assuming that during the oscillation the distribution functions of the two spin species behaves, in velocity space, as $f_{\uparrow \downarrow}\left(v_{x}, v_{y}, v_{z}\right)=f_{0}\left(v_{x}, v_{y}, v_{z} \pm u\right)$, where $f_{0}$ is the distribution function of each component at thermal equilibrium. This corresponds to a rigid displacement of the velocity Fermi distributions of the two spin components in opposite directions. One finds $\left\langle v_{z \uparrow}-v_{z \downarrow}\right\rangle=2 u$ and, by developing the integral (15) to first order in $u$, one can finally write

$$
\left\langle\left(v_{z \uparrow}-v_{z \downarrow}\right) I_{c o l l}\right\rangle=-\frac{\left\langle v_{z \uparrow}-v_{z \downarrow}\right\rangle}{\tau}
$$

where

$$
\begin{array}{r}
\frac{1}{\tau}=\frac{\sigma m^{7}}{24 \pi h^{6} N K_{B} T} \int d \mathbf{r} d \mathbf{v}_{1} d \mathbf{v}_{2} d \Omega|\mathbf{v}|\left(\mathbf{v}-\mathbf{v}^{\prime}\right)^{2} \times \\
\times f_{0}\left(\mathbf{v}_{1}\right) f_{0}\left(\mathbf{v}_{2}\right)\left(1-f_{0}\left(\mathbf{v}_{1}^{\prime}\right)\right)\left(1-f_{0}\left(\mathbf{v}_{2}^{\prime}\right)\right)
\end{array}
$$

defines the relevant relaxation time of the spin dipole oscillation. In (17) we have defined $\mathbf{v}=\mathbf{v}_{1}-\mathbf{v}_{2}$ and $\mathbf{v}^{\prime}=\mathbf{v}_{1}^{\prime}-\mathbf{v}_{2}^{\prime}$. As a consequence of (16) the equations of motion (13-14) for the spin dipole oscillation take the simple form of a damped harmonic oscillator. Looking for solutions of the form $e^{-i \omega t}$ the dispersion law is given by

$$
\omega=-\frac{1}{2 \tau}\left(i \pm \sqrt{4 \omega_{z}^{2} \tau^{2}-1}\right)
$$

showing that the oscillations become overdamped if $\omega_{z} \tau<1 / 2$.

The relaxation time (17) is easily evaluated at high temperature where the effects of Fermi statistics are negligible. In this case it takes the form

$$
\frac{1}{\tau_{c l}}=\frac{2}{3} \gamma_{c l}
$$

where $\gamma_{c l}=v_{t h} \sigma \rho(0) / 2$ is the classical collisional rate, $v_{t h}=\left(8 K_{B} T / \pi m\right)^{1 / 2}$ is the thermal velocity and $\rho(0)=$ $\rho_{\uparrow}(0)+\rho_{\downarrow}(0)$ is the central density of the gas. At temperatures smaller than the Fermi temperature $T_{F}=$ $(3 N)^{1 / 3} \hbar \omega_{h o} / K_{B}$ the effects of statistics become important and, for $T \rightarrow 0$, the relaxation time becomes larger and larger exhibiting the typical behaviour of Fermi systems. A similar behaviour has been recently shown to occur in the relaxation of the motion of a classical particle inside a degenerate trapped Fermi gas 19. By a proper change of the variables entering the collisional integral (17), the dimensionless quantity $\omega_{z} \tau$ can be written in the useful form (for oscillations along the radial direction one should consider the quantity $\left.\omega_{\perp} \tau=\omega_{z} \tau / \lambda\right)$

$$
\frac{1}{\omega_{z} \tau}=\frac{4}{3^{4 / 3} \pi} \lambda^{-2 / 3}\left(N^{1 / 3} \frac{a}{a_{h o}}\right)^{2} F\left(\frac{T}{T_{F}}\right)
$$

where $F(t)$ is a dimensionless function, sensitive to Fermi statistics, which determines the temperature dependence of the relaxation time. This function is plotted in Fig.(11). For large values of the reduced temperature $t=T / T_{F}$ it approaches the classical behaviour $1 / t$, while for $t$ smaller than 1 one observes, as expected, important deviations due to quantum effects. At very low temperatures the function $F(t)$ can also be calculated analytically and we find

$$
F(t)=8 \pi^{2} t^{2}
$$

Of course result (21) holds above the superfluid transition. The figure shows that the system will be in the collisionless regime both at sufficiently high and low temperatures. In the first case the gas is classical and collisions 
are rare because the density is very low. In the second one the gas is degenerate and collisions are rare because of Fermi statistics. The possibility for the system to reach the overdamped regime $\omega_{z} \tau<1 / 2$ depends in a crucial way on the value of the physical parameters entering (20). The maximum value of $F(t)$, reached at $t \sim 0.4$, is $\sim 1.6$ so that the condition for the existence of overdamping is given by $\lambda^{1 / 3} N^{1 / 3}|a| / a_{h o}>2.1$ (for the spin dipole oscillation along the radial axis the condition is instead $\left.\lambda^{-1 / 6} N^{1 / 3}|a| / a_{h o}>2.1\right)$. This condition should be easily achievable in the case of lithium. For example, choosing a spherical trap with $N=5 \times 10^{5}$ and $a / a_{h o}=-3 \times 10^{-2}$ one finds overdamping for $0.2<T / T_{F}<0.8$. For lower temperature the system will exhibit a transition to the collisionless regime where the spin oscillation is damped according to eq.(18). In the case of ${ }^{40} \mathrm{~K}$, where the value of the scattering length is much smaller, the system is expected to be always far from the hydrodynamic regime. Nevertheless an accurate investigation of the damping might point out the effects of Fermi statistics also in this case. In fact the damping of the spin oscillation is predicted to strongly decrease at low temperature in contrast to the classical behaviour. Notice that the above estimates are based on the unperturbed value $\omega_{z}\left(\omega_{\perp}\right)$ for the spin dipole frequency. At low temperature one can easily renormalize these values by including the mean field effect through eq.(12).

In conclusion, we have provided a systematic discussion of interactions effects on the propagation of collective oscillations in trapped Fermi gases. In the collisionless regime interactions renormalize the collective frequencies through mean field effects. The predicted shifts should be visible in experiments due to the high precision of frequency measurements. Collisions are responsible for the damping of the oscillations and their effect turns out to be very sensitive to Fermi statistics. Overdamped oscillations are predicted to occur in the case of the spin dipole oscillation and should be visible in lithium due to the high value of the scattering lenght. The present analysis can be naturally extended to include asymmetric configurations with different numbers of atoms in the two spin states $\left(N_{\uparrow} \neq N_{\downarrow}\right)$ and/or different trapping potentials $\left(V_{e x t}^{\uparrow} \neq V_{e x t}^{\downarrow}\right)$.

Useful discussions with D. Guéry-Odelin, H. Stoof and F. Zambelli are acknowledged.

[1] M. H. Anderson et al. , Science 269198 (1995); K. B. Davis et al. , Phys. Rev. Lett. 75, 3969 (1995); C. C. Bradley et al. , Phys. Rev. Lett 78, 985 (1997); see also Phys. Rev. Lett. 75, 3969 (1995)

[2] F. Dalfovo, S. Giorgini, L. P. Pitaevskii and S. Stringari, Rev. of Mod. Phys. 71463 (1999)

[3] D. S. Jin et al., Phys. Rev. Lett. 77, 420 (1996); M.-
O. Mewes et al., Phys. Rev. Lett. 77, 988 (1996); D. M. Stamper-Kurn, H.-J. Miesner, S. Inouye, M. R. Andrews, W. Ketterle, Phys. Rev. Lett. 81, 500 (1998)

[4] M. Houbiers, R. Ferwerda, H. T. C. Stoof, W. I. McAlexander, C. A. Sackett, R. G. Hulet, Phys. Rev. A 56, 4864 (1997)

[5] G. M. Bruun, K. Burnett, Phys. Rev. A 58, 2427 (1998)

[6] K. Mølmer, Phys. Rev. Lett. 80, 3419 (1998)

[7] M. Amoruso, A. Minguzzi, S. Stringari, M. P. Tosi, L. Vichi, Eur. Phys. J. D 4, 261 (1998)

[8] M. Prevedelli et al. , Phys. Rev. A 59, 886 (1999); B. DeMarco, J. L. Bohn, J. P. Burke Jr. M. Holland, D. S. Jin, cond-mat/9812350

[9] M. A. Baranov, Yu. Kagan, M. Yu. Kagan, JETP Lett. 64, 301 (1996)

[10] H. T. C. Stoof, M. Houbiers, C. A. Sackett, R. G. Hulet, Phys. Rev. Lett. 7610 (1996)

[11] M. A. Baranov, D. S. Petrov, cond-mat/9901108

[12] D. Pines, P. Noziéres The Theory of Quantum Liquids Vol. I, Addison-Wesley

[13] S. Stringari, J. Low Temp. Phys. 57, 307 (1984); O. Bohigas, A. M. Lane, J. Martorell, Phys. Rep. 51, 267 (1979); E. Lipparini and S. Stringari, Phys. Rep. 175 (1989) 103261

[14] S. Stringari, Phys. Rev. Lett. 77, 2360 (1996)

[15] I. F. Silvera, Physica 109 \& 110B 1499 (1982); D. A. Butts, D. S. Rokhsar, Phys. Rev. A 554346 (1997)

[16] A. Bohr, B. R. Mottelson, Nuclear structure Vol. II, Benjamin

[17] M. Amoruso, I. Meccoli, A. Minguzzi, M. P. Tosi, accepted for pubblication in Eur. Phys. J. D

[18] A. Griffin, Wen-Chin Wu, S. Stringari, Phys. Rev. Lett. 781838 (1997)

[19] G. Ferrari, cond-mat/990416, accepted for pubblication in Phys. Rev. A, rapid communications

[20] D. Guéry-Odelin, F. Zambelli, J. Dalibard, S. Stringari, preprint cond-mat/9904409

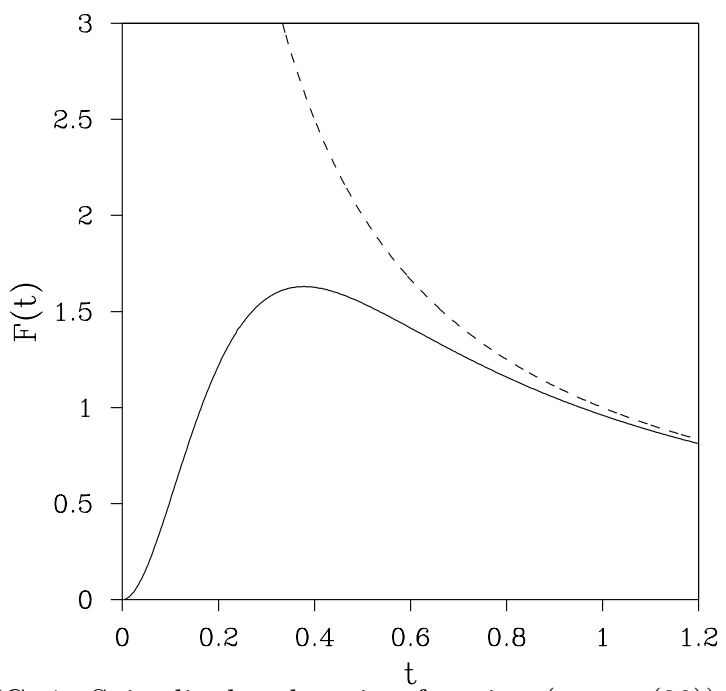

FIG. 1. Spin dipole relaxation function (see eq. 200) as a function of the reduced temperature $t=T / T_{F}$. The classical prediction $1 / t$ (dahsed line) is also shown. 